\author{
A Thesis by \\ Yibin Wang \\ Master of Art, Wichita State University, 2011 \\ Bachelor of Science, University of Qingdao Science and Technology, 2007 \\ Submitted to the Department of Industrial and Manufacturing Engineering \\ and the faculty of the Graduate School of \\ Wichita State University \\ in partial fulfillment of \\ the requirements for the degree of \\ Master of Science
}

May 2013 
(C) Copyright 2013 by Yibin Wang

All Rights Reserved 


\section{COST BENEFIT ANALYSIS OF CONDITION MONITORING SYSTEMS FOR OPTIMAL MAINTENANCE DECISION MAKING}

The following faculty members have examined the final copy of this thesis for form and content, and recommend that it be accepted in partial fulfillment of the requirements for the degree of Master of Science with a major in Electrical Engineering.

Pingfeng Wang, Committee Chair

Janet Twomey, Committee Member

Jen-Chi Cheng, Committee Member 


\section{DEDICATION}

To my family and friends 


\section{ACKNOWLEDGEMENT}

I would first and foremost like to express my deep gratitude to my adviser, Dr. Pingfeng Wang. He patiently guided me through the research project, never accepting less than my best efforts. His wisdom, knowledge and commitment to the highest standards inspired and motivated me.

I am also indebted to my project defense committee members, Dr. Janet Twomey and Dr. Jen-Chi Cheng for their time, support and helpful comments.

Special thanks go to all of my lab members in the Reliability Engineering Automation Laboratory at Wichita State University, for sharing innumerous days and nights in the lab and for their encouragements and support when I was in need.

Finally, I heartily appreciate my family who has given meaning to all the effort I have done. This thesis could not have been done without their unwavering love and support. 


\begin{abstract}
Tremendous advances in high performance sensing and signal processing technology enable the development of condition monitoring systems (CMS) for complex engineered systems to detect, diagnose, and predict the system-wide effects of failure events. Although employing CMS in preventing catastrophic system failures and reducing the operation and maintenance (O\&M) costs have been acknowledged, the cost and benefit of CMS have not been well studied and further the advantages of CMS have not been fully recognized for the optimal maintenance decision making, mainly due to the lack of valid theoretical modeling addressing the interrelationship between the CMS effectiveness and system downtime due to system failures. In this study, a Poisson Process model will be developed for the modeling of occurrence of the system-wide failure events and study the potential benefits provided by the CMS in preventing these failure events. With the developed Poisson process model, the cost benefit analysis (CBA) will then be implemented by considering the CMS system reliability and costs varying with its failure detection effectiveness presented by the probabilistic detectability measure. Facilitated by CBA of the CMS, break-even points (BEP) between expected lifecycle benefits and the required CMS detectability level can be found to select optimal CMS for different system failure modes. Moreover, with the help of the CBA results, optimal maintenance strategies can be determined to minimize the O\&M costs. The presented CBA methodology for the CMS systems will be demonstrated with an aircraft maintenance case study and the efficacy will be validated.
\end{abstract}




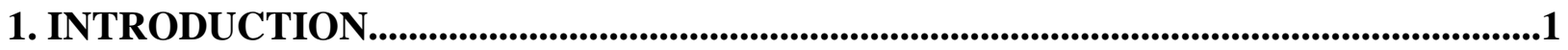

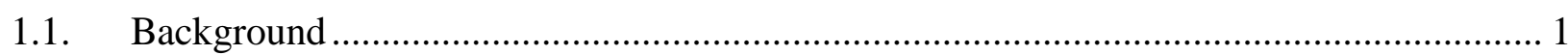

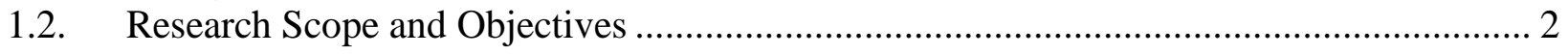

\section{LITERATURE REVIEW}

2.1. Wind Turbine Condition Monitoring Technique ............................................................ 4

2.1.1 Vibration Analysis ........................................................................................ 4

2.1.2. Electrical Signature Analysis .......................................................................... 5

2.1.3. Acoustic Analysis ..................................................................................... 5

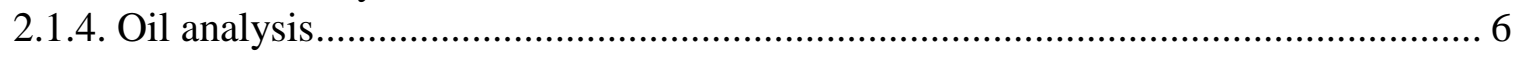

2.2. Cost Benefit Analysis of Condition Monitoring System .................................................... 6

2.3. Maintenance Strategies ................................................................................... 7

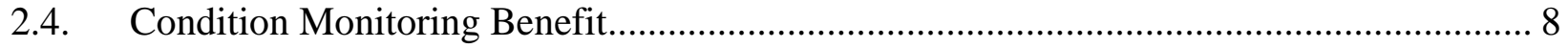

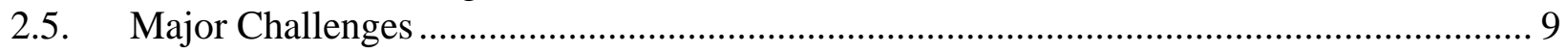

2.6. Technical Needs for Improvement........................................................................... 9

3. MODELING OF DESCRETE SYSTEM FAILURE EVENTS .............................................10

3.1. Constant Failure Rate Model and the Homogeneous Poisson Process .............................. 10

3.2. Time-variant Failure Rate Model and Non-Homogeneous Poisson Process ..................... 11

4. COST BENEFIT ANALYSIS OF CMS FOR MAINTENANCE .........................................13

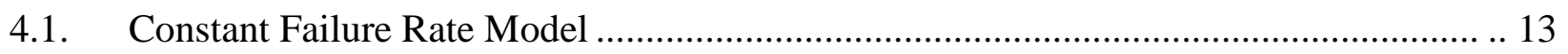

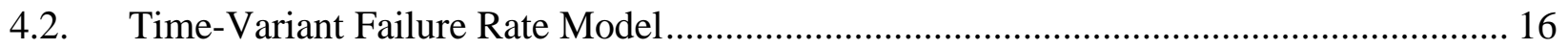

4.3. Multiple Failure Modes with Multiple CMS Systems............................... 18

4.4. Multiple Failure Modes Considering CMS System Failures..........................21

5. COST BENEFIT ANALYSIS CASE STUDY ........................................................................22 


\section{TABLE OF CONTENTS}

Chapter

6. CONCLUSION ………...............................................................................................................................25

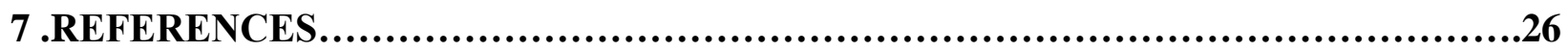




\section{LIST OF TABLES}

Table

Page

1. Parameters Of CBA For CMS With A Constant Failure Rate Model

2. Parameters Of CBA For CMS With A Time-Variant Failure Rate Model

3. Model C Parameters

4. Model Parameter For the Case Study 


\section{LIST OF FIGURES}

Figure

Page

1. Distribution of Number of Failures 15

2. CBA for CMS Results 15

3. Distribution of Number of Failures 17

4. CBA for CMS Results 17

5. Multiple CMS Multiple Failure Mode Correspondence 18

6. CBA For CMS Results With Two Time-Variant Failure Mode 20

7. Number of Failure Distributions for Failure Modes and CMS systems 23

8. Expected Cost Savings with respect to Detectability Levels from CMS Systems 24

9. Contour of the Overall Cost Saving with respect to $D_{11}$ and $D_{21}$ 


\section{LIST OF NOMENCLATURE}

$\begin{array}{ll}\lambda & \text { Failure rate } \\ B E P & \text { Break even point } \\ C_{m} & \text { Maintenance cost } \\ C_{f} & \text { Failure cost } \\ C_{f} & \text { Failure cost } \\ C_{c m s} & \text { Condition monitoring system cost } \\ C_{s} & \text { Cost benefits } \\ C M S & \text { Condition monitoring system } \\ \mathrm{D}_{t} & \text { Detectability level } \\ E(.) & \text { Expected value } \\ f(x) & \text { Probability density function } \\ p_{f} & \text { Probability of failure } \\ H P P & \text { Homogeneous Poisson process } \\ N H P P & \text { Non-homogeneous Poisson process } \\ P_{n}(t) & \text { Stochastic process } \\ & \end{array}$




\section{CHAPTER 1}

\section{INTRODUCTION}

\subsection{Background}

Effective system condition monitoring, which monitors a parameter of condition in system components or subsystem so that a significant change is indicative of a developing failure, provides multifarious benefits such as improved safety, improved reliability and reduced costs for operation and maintenance of complex engineered systems [1-4]. Maintenance and lifecycle management is one area that is positioned to significantly benefit in this regard due to the pervasive nature of design and maintenance activities throughout the manufacturing and service sectors. Maintenance and life-cycle management activities constitute a large portion of overhead costs in many industries [3]. These costs are likely to increase due to the rising competition in today's global economy. In the manufacturing and service sectors, unexpected breakdowns can be prohibitively expensive since they immediately result in lost production, failed shipping schedules, and poor customer satisfaction. In order to reduce and possibly eliminate such problems, it is necessary to accurately assess the current state of system degradation through effective health diagnostics. Two major research areas have tried to address these challenges: reliability and condition monitoring. Although reliability and condition monitoring are seemingly related, reliability focuses on population-wide characteristics while condition monitoring deals with component-specific properties. Furthermore, both of fields' researches have evolved separately. Research in the reliability area can be broadly classified into two subcategories. One category focuses on quantification of reliability and statistical analysis of run-to-failure data, such as [3-5]while the deals with the development of physical-based models (e.g., fatigue, wear, 
and corrosion). In contrast, condition monitoring research uses sensory information from functioning system to access their degradation states. Continuous monitoring of current health states through multi sensors notifies the performance degradation of component at both early and advanced stages of damage. Real time diagnosis of sensory data acquired from the overall sensor network and data analysis helps to make crucial decisions on significant improvement over wide range of applications. Some of the successful applications of condition monitoring include condition monitoring of bearings [6,7], machine tools [8], transformers [9], engines [10], and turbines [11] among many others.

\subsection{Research Scope and Objectives}

Despite the importance of the real time condition monitoring and various successful application of condition monitoring techniques, the design and implementation of effective condition monitoring system (CMS) to meet desired performance requirements could be very expensive and require ample knowledge for specific application fields and understanding of system failure modes as well as indicative failure phenomena. This thesis presents a novel cost benefit analysis approach for condition monitoring system, in which Non-Homogeneous Poisson Process (NHPP) model is developed for the modeling system-wide failure events and study the potential benefits provided by the CMS in preventing these failure events. With the developed NHPP model, the cost benefit analysis (CBA) will then be implemented by considering CMS costs varying with failure detection effectiveness presented by the probabilistic detectability measure. Facilitated by CBA of the CMS, break-even points (BEP) between expected useful life benefits and the required CMS detectability level can be found to select the optimal CMS for different system failure modes. Moreover, with the help of the CBA results, optimal maintenance strategies can be determined to minimize the O\&M costs. The presented CBA methodology for 
the CMS will be demonstrated with an aircraft maintenance case study and the efficacy will be validated. The remaining of the thesis is organized as follows: Section 2 explains the modeling of discrete system failure events over time with Poisson Process models; Section 3 derives the expected benefits of CMS for system maintenance; Section 4 details the proposed cost benefit analysis approach; Section 5 demonstrates the proposed approach with one case study. A brief summary of presented work and potential future studies is provided in Section 6. 


\section{CHAPTER 2}

\section{LITERATURE REVIEW}

This section gives a brief overview of the various journal papers, text books, conference papers, technical publications related to the project. The related work under this topic includes: (1) wind turbine condition monitoring technique; (2) Cost benefit analysis of condition monitoring system; (3) Life cycle cost analysis tools ; (4) Maintenance strategies (5) Condition maintenance benefits; (6) Major Challenges; (7) Technical needs for improvement.

\subsection{Wind Turbine Condition Monitoring Technique}

\subsubsection{Vibration Analysis}

Vibration analysis is the most common technique and provides the early fault detection in rotating components of the wind turbine condition monitoring and it can be used in different components such as the gearbox, generator and drive train. In this way, the vibration sensors will be installed on the components[12]. Therefore, it is quite expensive to adopt the vibration based monitoring system[13]. But the accuracy of vibration analysis for the health monitoring has been proven by different literatures. With the vibration analysis, the bearing defects, imbalance and misalignment can be identified.

Broad band method or spectral line analyses are the output signals analyzing methods. In the broad band analysis, the output signals can be utilized to calculate the necessary parameters such as root mean square, peak value, or kurtosis. Comparing the differences between the initial parameters and the current value of the parameters, the failure can be estimated and observed. The spectral lines analysis is based on the physical properties of the components since components show various frequencies of vibration signatures. 
Gearbox is one of the most important units in the wind turbine and the vibration analysis has been introduced for detecting the failure of the gearbox. Yang et al. developed a back propagation neural network (BPNN) base framework to identify the actual fault status of the gearbox[14]. Huang et al. implements the wavelet neural networks on vibration fault diagnosis for wind turbine gearbox in 2008[15]. By using this WNN method, the accuracy of the diagnostic is effective and shows better results than the BPNN method.

\subsubsection{Electrical Signature Analysis}

Electrical signature analysis is a process of acquiring the current and voltage signals and performing the analysis to identify different damage growth. Under this approach, electrical sensors are installed in generators of the wind turbine to analyze the current and voltage from the generators[16]. In this method, there is no need to stop the wind turbine and the operator can monitor the signal on the remote base. Also since this technology has been in the market for over 20 years, it is well recognized by industries. The advantages of electrical signature analysis are easy access since the current output is already available, reducing the number of sensors by comparing with the vibration analysis and oil analysis, and cheaper cost since it uses the electrical measurements instead of the mechanical measurements[12]. There are several different signals processing method to perform the electrical analysis. Spectral methods and wavelet analysis are the most influence ones.

\subsubsection{Acoustic Analysis}

Acoustic analysis is quite close to vibration analysis. However, there is a basic difference between these two analysis methods. For the vibration analysis, the sensors are installed on the components and the local vibration can be detected by the sensors. For the acoustic analysis, the sensors which are attached on the components by strong glue are acquiring signals by listening to 
the components[10]. Gearbox and bearing have been successfully diagnostic by using the acoustic analysis technique[17]. Another literature shows the wind turbine blades certification tests can be carried out by the acoustic analysis[18]. The most important advantage of the acoustic analysis is, it can detect the faults in their early stage. Also the acoustic analysis is usually used for the low speed operation components.

\subsubsection{Oil analysis}

Oil analysis is usually carried out when the system is offline. Oil analysis on wind turbines is an effective tool to assess lubricant condition, contamination, and mechanical wear. Therefore, the oil analysis can give some valuable information about the health condition of rotating components. The disadvantage of the oil analysis is, it can only perform when the oil is in the closed loop of the supply system and quite expensive to operate when the system is online. According to the maintenance manual, the gearbox oil should be replaced every four year and this could be done at least five times during the wind turbine life time. But when applied the oil analysis, the oil change may only need to be changed every seven year[19]. This value information could help the wind farm save lots of money during the operation and maintenance activities.

\subsection{Cost Benefit Analysis of Condition Monitoring System}

In cost benefit analysis of condition monitoring system literature, the cost benefit of installing the condition monitoring system on the wind turbine has been widely simulated using either probabilistic methods or deterministic methods. In 2007, a stochastic simulation research was done by comparing the advantage and disadvantage between the schedule maintenance and the condition based maintenance[20]. The cost benefit has been further invested in this thesis and the potential value of adopting condition monitoring system is demonstrated in the case study. 
The final results show the wind turbine gearbox will have a $\$ 40,000$ saving with the help of condition monitoring system. With some uncertainties exist, the accuracy of the CMS is not well understood and the reliability of CMS itself has not been investigate. In 2010, the author Francois presents a stochastic simulation model to analyze the economics benefit of using condition monitoring systems under some deterministic input parameters of the wind turbine system. The life cycle costs of different maintenance plans are shown in this thesis. The plan with CMS is performing much better than without CMS. The CMS system has potential economic benefits to the wind turbine failure prevention[21].

\subsection{Maintenance Strategies}

Maintenance activities are necessary for retaining or restoring a component or system to its operating condition and extend its working lifetime. The maintenance activities can be classified into two categories: reactive maintenance and proactivity maintenance. The reactive maintenance includes the corrective maintenance which performs the maintenance activities after the failure event happen. The corrective maintenance is always associated with the replacement activities, emergency or unplanned activities. Since reactive maintenance is not well planned, the proactivity will come to the place. The proactivity maintenance includes the scheduled maintenance and condition based maintenance. The scheduled maintenance is required to inspect the system every certain time period and replace the components when it reaches its replacement criteria in the same visiting time. The condition based maintenance includes the online monitoring and offline monitoring maintenance. Online monitoring maintenance is continuously monitoring the health condition of the system and performing the online health condition analyze. It is normally equipped with an online accessing device [22]. Wiggelinkhuizen et al. believe three requirements are associated with the condition monitoring technique. Requirements are the 
detection of failure mechanism, detection on time, and measureable criteria [19].Offline monitoring maintenance is usually performed by holding a health detecting devices like infrared thermograph or done by the oil analysis, vibration analysis [23]. Each type of maintenance strategy has its own advantages and disadvantages. For the reactive maintenance, the advantage of it is maximize the life span of the components and the drawbacks of it are non-predictive failure happen, no spare parts on hand, or high chance to cause the consequential damages by the failure parts. For the active maintenance, the advantages are predicting failure in the early stage, lower downtime and production loss, or plan well on the inventory reserve. The limits of this maintenance are, it is expensive to have the CMS devices and how actuate the sensors can tell you. Also the components will not be used for their maximum lifetime[12].

\subsection{Condition Monitoring Benefit}

System failures could happen within machinery regardless of even the most well planned scheduled maintenance with often-unnoticed faults leading to unexpected failures before performing the next regular maintenance. Condition monitoring equips the operators in control of the system health condition and allow them to detect the failure in the early stage, help operators avoid unscheduled breakdown and give enough time for operators to prepare the inventory. Condition monitoring measures the health state of the system by continuously monitoring and online embedded data analysis.

Efficient and continuous production and has the proven potential to save thousands of dollar in additional damage such as production loss and failure maintenance. Condition Monitoring is a sound, proactivity maintenance strategy that provides early indication of preventative faults. In 2007, a life cycle cost analysis for one wind farm maintenance activities have been studied. Case studies show condition monitoring can reduce the unexpected break 
down and increase the productivity during the wind farm operating time, especially for the offshore wind farm maintenance planning [20].

\subsection{Major Challenges}

Due to the nature of the failure, nobody can know exactly what failure will happen at any given time point. Scheduled maintenance in large scale system may miss many failures and this will increase large amount of capital costs to have the spare parts in hand, unscheduled labor cost, production loss, etc. How to make a good operation and maintenance plan becomes a key point in wind farm operation. Condition monitoring system may have a solution for this, but the question to ask when evaluating the CMS is whether the cost associated with the CMS can be validated through the usefulness of the CMS.

\subsection{Technical Needs for Improvement}

Most of the condition monitoring related literatures assume the detectability of the CMS is perfect and with no false alarm. A stochastic evaluation of the LCC simulation has been carried out by [21] and the author assumed the detection rate of CMS is $90 \%$. But in reality, CMS will not detect all the failure events in the practical world. Therefore, building a model has the ability to investigate the real economic benefits of CMS becomes the top priority in this field study. Also the reliability of CMS and LCC of the CMS should be targeted at and this part of information must be taken into the consideration when making the operation and maintenance plan. 


\section{CHAPTER 3}

\section{MODELING OF DESCRETE SYSTEM FAILURE EVENTS}

For engineered system, occurrence of discrete system failure events can be modeled by reliability in a statistical manner, in which reliability implies the ability of a system or component to perform its required functions under stated conditions for a specified period of time. Mathematically, this may be expressed as,

$$
R(t)=\operatorname{Pr}\{T>t\}=\int_{t}^{+\infty} f(x) d x
$$

Where $\mathrm{f}(\mathrm{x})$ is the failure probability density function and $\mathrm{t}$ is the length of the period of time which is assumed to start from time zero. Considering the design life of a system, the failure rate is an important concept in modeling the discrete system failure events, which can be defined as the total number of failures within an item population, divided by the total time expended by that population, during a particular measurement interval under stated conditions. It is often denoted by the $\lambda(\mathrm{t})$ and is important in reliability engineering. Mathematically, this may be expressed as,

$$
\lambda(t)=\frac{f(t)}{R(t)}
$$

Failure rate of a system or component could be constant over time or time-variant, depending on different failure modes in consideration. Time variant failure rate indicates that failure frequency of a system depends on time, with the rate varying over the life cycle of the system. In the following of this session, the constant failure rate model and time-variant failure rate model will be explained and the Poisson process will be introduced in modeling the occurrence of discrete system failure events over time.

\subsection{Constant Failure Rate Model and the Homogeneous Poisson Process}


Starting from the simple failure mode which the failure distribution $f(t)$ follows exponential distribution as

$$
f(t)=\lambda e^{-\lambda t}
$$

For a constant failure rate model, failure events of a system or component will occur randomly over the life of the system. Then the probability of having total number of $\mathrm{n}$ failures within time $t, P n(t)$, can be expressed by the homogeneous Poisson processes, as:

$$
P_{n}(t)=\frac{e^{-\lambda t}(\lambda t)^{n}}{n !}
$$

\subsection{Time-variant Failure Rate Model and Non-Homogeneous Poisson Process}

The failure rate of a system usually depends on time, with the rate varying over the life of the system. For example, an automobile's failure rate in its fifth year of service may be many times greater than its failure rate during its first year of service. One does not expect to replace an exhaust pipe, overhaul the brakes, or have major transmission problems in a new vehicle. For the simplicity of explanation, we assume that the failure distribution for a time dependent failure mode follows the Weibull distribution. Weibull distribution is a commonly used reliability distribution to model the time to failure of the components or the systems. If the failure distribution follows a Weibull distribution, with the scale parameter and shape parameter are $\theta$ and $\beta$ separately, the failure rate $(\lambda)$ of the Weibull distribution can be expressed as:

$$
\lambda(t)=\frac{\beta}{\theta}\left(\frac{t}{\theta}\right)^{\beta-1}
$$

With this failure rate function, the probability of having $\mathrm{n}$ number of failures during the design life $\mathrm{L}$ of a system follows a Non-Homogenous Poisson Process and can be evaluated as: 


$$
P_{n}(t)=\frac{e^{-\int_{0}^{L} \lambda(t) d t}\left(\int_{0}^{L} \lambda(t) d t\right)^{n}}{n !}
$$




\section{CHAPTER 4}

\section{COST BENEFIT ANALYSIS OF CMS FOR MAINTENANCE}

In this section, the cost benefit analyses of employing a CMS system for system failure modes that are characterized by either a constant failure rate model or a time-variant failure rate model are presented. In the following discussion, we assume that a CMS system with a cost of $\mathrm{C}_{\mathrm{CMS}}$ has the capability to detect the failure mode before the occurrence of the actual failure event at a certain probability level, which can be expressed by a detectability measure as noted by the $D_{t}$ that falls within the range between 0 and 1 . Also, it is assumed that once a potential failure event is detected, appropriate maintenance activities would return the system to as good as new condition at a maintenance cost of $\mathrm{C}_{\mathrm{m}}$. If the failure event occurred, the failure cost of a system for the considered failure mode will be $C_{f}$, where $C_{f} \gg C_{m}$. The failure cost could include the component cost, logistic cost, labor cost and other collateral or consequential damage cost due to the failure. With the notations introduced above, the cost benefit analysis to be carried out in the remaining of this section aims to find out the expected cost savings, $\mathrm{C}_{\mathrm{s}}$, for maintenance within the design life of a system while adopting a CMS system.

\subsection{Constant Failure Rate Model}

For a failure mode that follows constant failure rate model, the number of failures that could occur during the design life of a system follows a homogeneous Poisson process, as discussed in section 3.1. Assuming that there will be totally n number of discrete failure events occurred, by employing a CMS system with a detectability level of $\mathrm{D}_{t}$ at a cost of $\mathrm{C}_{\mathrm{CMS}}$, the benefits in terms of maintenance cost saving can be expressed as

$$
\begin{aligned}
C_{S}(n) & =n \cdot C_{2}-n \cdot D_{t} \cdot C_{1}-n \cdot\left(1-D_{t}\right) \cdot C_{2}-C_{C M S} \\
& =n \cdot D_{t}\left(C_{2}-C_{1}\right)-C_{C M S}
\end{aligned}
$$


As the total number of discrete failure events, $n$, over the design life of a system is a random variable as described by Eq.(4), it is impossible for the decision maker to know exactly how many failure events would occur. It is more convenient to to carry out the expected cost savings while considering the probability of different number of failure events by taking the following expected benefit in terms of the cost saving function, which can be expressed as

$$
E\left[C_{S}\right]=\sum_{n=1}^{\infty} \frac{e^{-\lambda t}(\lambda t)^{n}}{n !} \cdot\left\{n \cdot D_{t}\left(C_{2}-C_{1}\right)-C_{C M S}\right\}
$$

Moreover, while considering the cost and the detectability metric of a CMS system, we are able to determine the break event point (BEP) of the CMS system cost, ${ }^{{ }_{C M S}}$ by setting the cost saving equals to zero in Eq.(7), as

$$
\hat{C}_{C M S}=n \cdot D_{t}\left(C_{2}-C_{1}\right)
$$

With the BEP of the CMS system cost, the decision could be made to adopt a CMS system for a certain system failure mode will save lifecycle maintenance costs at the system operational stage.

The following example will demonstrate the cost benefit analysis with constant failure rate models. As you can see from the table below, the design life (L) and failure rate of this example system is 20 years and 0.333 times per year, respectively. The regular maintenance cost $\mathrm{Cm}$ is $\$ 5,000$ and the failure cost $\mathrm{C}_{\mathrm{f}}$ is $\$ 20,000$. The cost for the CMS system, $\mathrm{C}_{\mathrm{cms}}$, is $\$ 15,000$. With the given parameter values in Table 1, the cost benefit analysis is carried out and the results are shown in Fig. (1) and Fig. (2). As shown in Fig. (1), the range of total number of failure events over the design life L varies from 1 to 15 , with high on probabilities between 5 and 10 . Figure. (2) represents the expected benefits with respect to the detectability level of the CMS system given system cost $\mathrm{C}_{\text {cms. }}$ The figure indicates that CMS system detectability level must be 
no less than $15 \%$ in order to result in a maintenance cost savings that are greater than the cost of CMS system itself.

Table 1. Parameters of CBA for CMS with a Constant Failure Rate Model

\begin{tabular}{lllll}
\hline \hline$\lambda$ & $C_{m}$ & $C_{f}$ & $C_{C M S}$ & $L$ \\
\hline $0.333 /$ year & 5,000 & 20,000 & 15,000 & 20 years \\
\hline
\end{tabular}

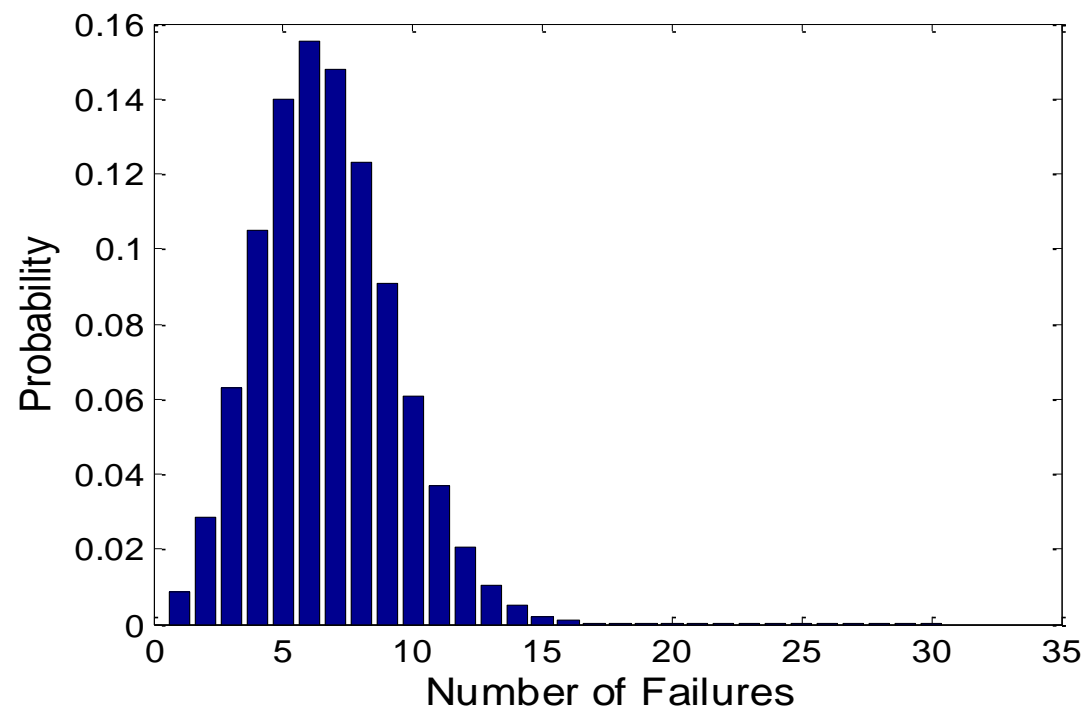

Figure 1. Distribution of Number of Failures $n$ over lifetime L.

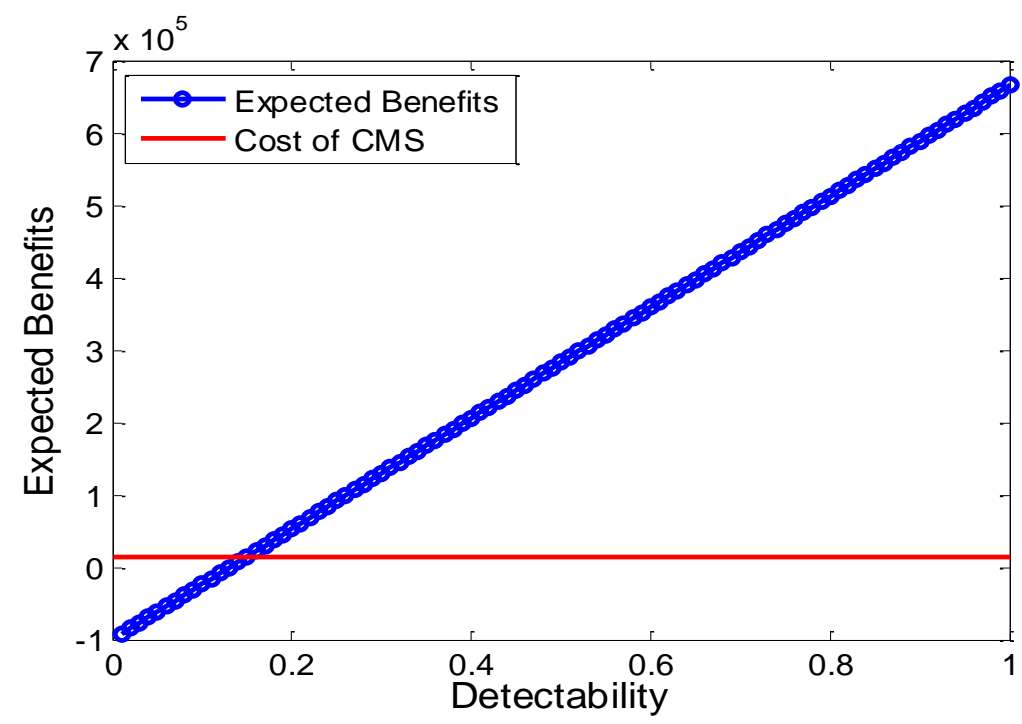

Figure 2.CBA for CMS Results 


\subsection{Time-Variant Failure Rate Model}

For a failure mode that follows time-variant failure rate model, the number of failures that could occur during the design life of a system follows a non-homogeneous Poisson process, as discussed in the previous section. Assuming that the failure distribution follows two parameter Weibull distribution and there are n number of descrete failure events occurred, by employing a CMS system with a detectability level of Dt at a cost of CCMS, the benefits in terms of maintenance cost saving can be expressed as

$$
\begin{aligned}
C_{S}(n) & =n \cdot C_{2}-n \cdot D_{t} \cdot C_{1}-n \cdot\left(1-D_{t}\right) \cdot C_{2}-C_{C M S} \\
& =n \cdot D_{t}\left(C_{2}-C_{1}\right)-C_{C M S}
\end{aligned}
$$

As the total number of descrete failure events, $n$, over the design life of a system is a random variable according to Eq.(6), it is convenient to carry out the expected cost savings while considering the probability of different number of failure events, which can be expressed as

$$
E\left[C_{S}\right]=\sum_{n=1}^{\infty} \frac{e^{-\int_{0}^{L} \lambda(t) d t}\left(\int_{0}^{L} \lambda(t) d t\right)^{n}}{n !} \cdot\left\{n \cdot D_{t}\left(C_{2}-C_{1}\right)-C_{C M S}\right\}
$$

The following example will demonstrate the cost benefit analysis with time-variant failure rate models. As discussed in the previous section, total number of failure events of the system follows a NHPP with Weibull distribution shape and scale parameter $\beta$ and $\theta$ are 0.5 and 3, respectively. The design life $(L)$ is kept as 20 years. The regular maintenance cost $C_{m}$ is $\$ 5,000$ and the failure cost $C_{f}$ is $\$ 20,000$. The cost for the CMS system, $C_{C M S}$, is $\$ 15,000$. With the parameter values indicated in Table 2, the cost benefit analysis is carried out and the results are shown in Fig. (3) and Fig. (4). As shown in Fig. (3), the range of total number of failure events over the design life $L$ varies from 0 to 14, with high probabilities within the range between 0 and 5. Figure. (4) represents the expected maintenance cost savings with respect to the detectability level of the CMS system given that the system cost $C_{C M S}$, which indicates that CMS system detectability level must be no less than $37 \%$ in order to result in a maintenance cost saving that is greater than the cost of CMS system itself. 
Table 2. . Parameters of CBA for CMS with a Time-Variant Failure Rate Model

\begin{tabular}{llllll}
\hline \hline$\beta$ & $\theta$ & $C_{m}$ & $C_{f}$ & $C_{C M S}$ & $L$ \\
\hline 0.5 & 3 & 5,000 & 20,000 & 15,000 & 20 \\
\hline
\end{tabular}

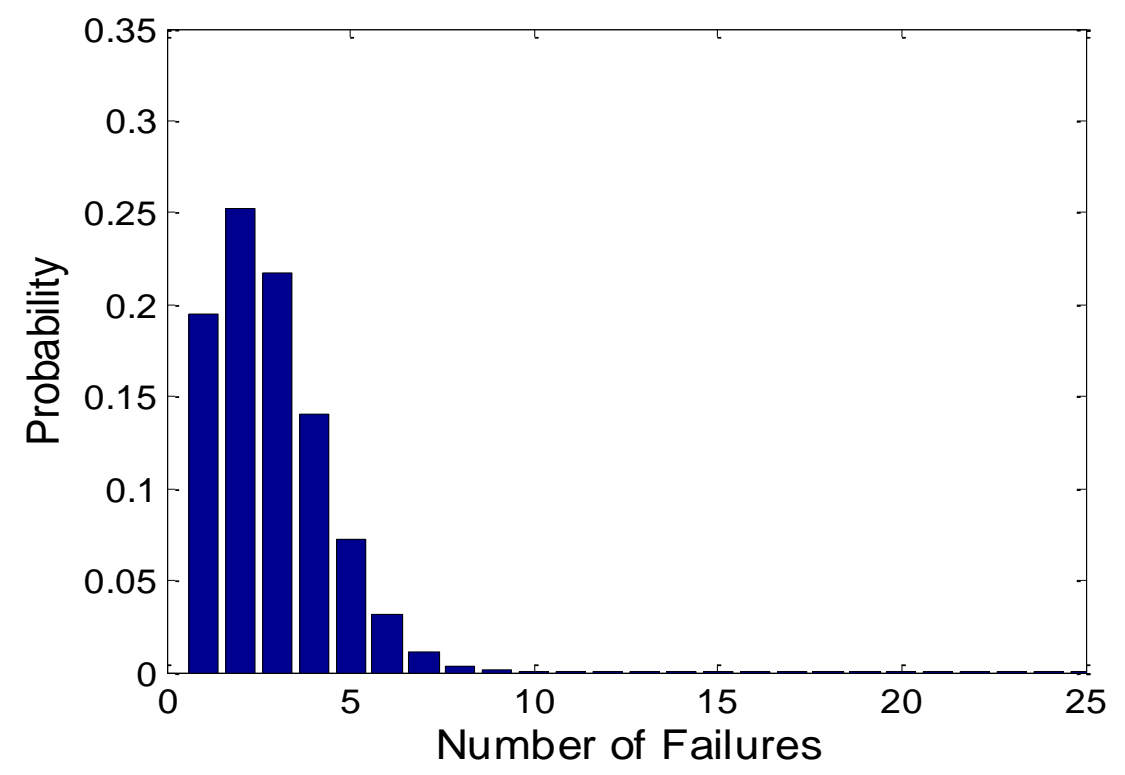

Figure 3. Distribution of Number of Failures

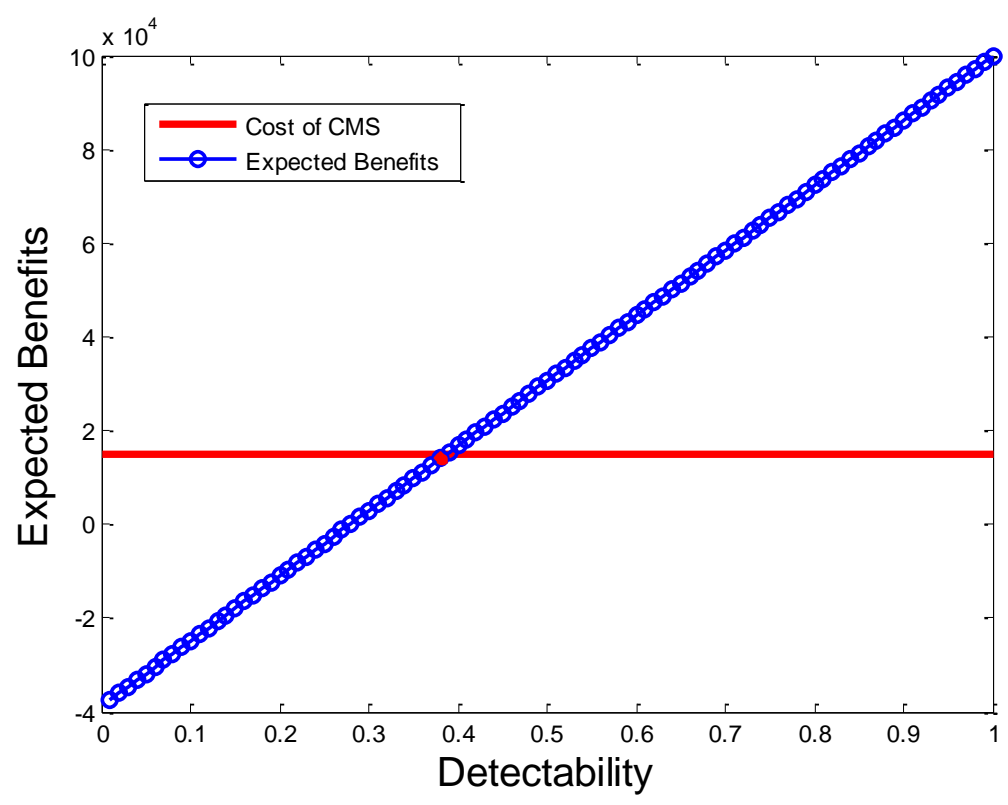

Figure 4. CBA for CMS Results 


\subsection{Multiple Failure Modes with Multiple CMS Systems}

In this subsection, our objective is to investigate the cost benefit analysis problem that involves multiple failure modes with different failure rate functions and multiple condition monitoring systems. Let $k$ be the total number of condition monitoring systems being employed, $n$ be the total number of failure modes being considered and $\lambda_{j}(\mathrm{t})$ be the failure rate function for the $i^{\text {th }}$ failure mode, where $j=1, \ldots, n$. The detectability of the $i^{\text {th }}$ CMS $(i=1 \ldots k)$ to the $j^{\text {th }}$ failure mode is denoted with $D_{i j}$, as shown in the Fig. (5) by the multiple CMS multiple failure modes correspondence diagram. Assuming the independency between different failure modes and the CMS systems, the detectability for the $\mathrm{j}^{\text {th }}$ failure mode, $D_{j}$, considering all the CMS systems can be obtained as:

$$
D_{j}=1-\prod_{i=1}^{k}\left(1-D_{i j}\right)
$$

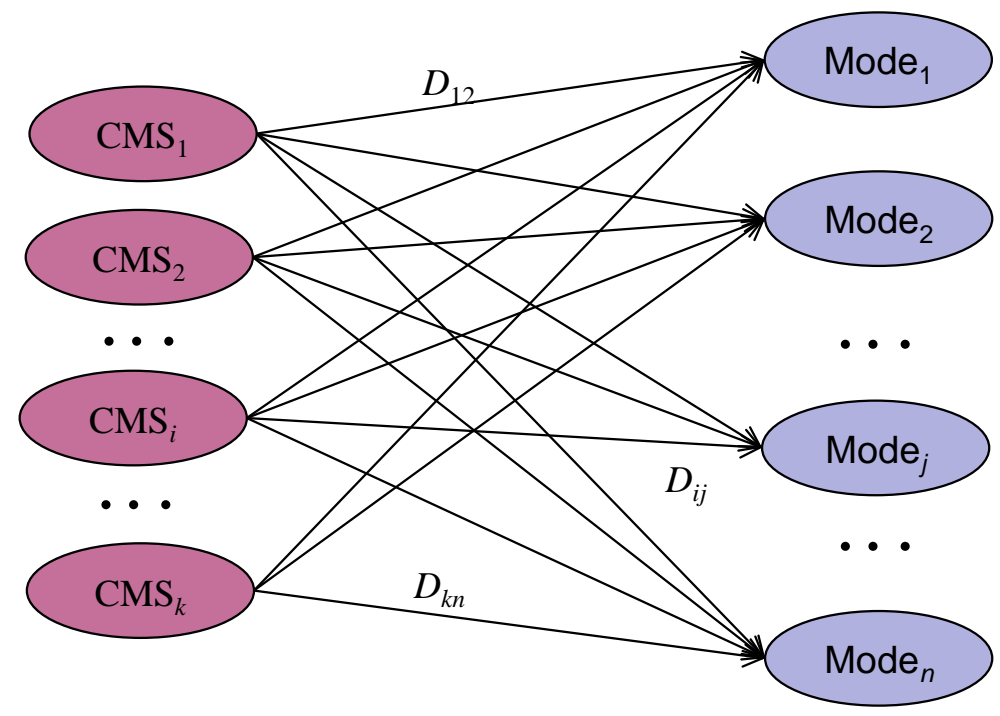

Figure 5. Multiple CMS Multiple Failure Mode Correspondence 
Based on the previous discussion, there are $n_{j}$ number of discrete failure events occurred for the $j^{\text {th }}$ failure mode, employing multiple CMS systems with an overall detectability level of $D_{j}$, the benefits in terms of maintenance cost saving without considering the cost for the CMS systems can be expressed as

$$
C_{S}^{j}\left(n_{j}\right)=n_{j} \cdot C_{2}^{j}-n_{j} \cdot D_{j} \cdot C_{2}^{j}-n \cdot\left(1-D_{j}\right) \cdot C_{2}^{j}=n_{j} \cdot D_{j}\left(C_{2}^{j}-C_{1}^{j}\right)
$$

As the total number of discrete failure events, $n_{j}$, over the design life of a system is a random variable as described by Eq.(6), it is convenient to carry out the expected cost savings while considering the probability of different number of failure events, which can be expressed as

$$
E\left[C_{S}^{j}\right]=\sum_{n_{j}=1}^{\infty} \frac{e^{-\int_{0}^{L} \lambda_{j}(t) d t}\left(\int_{0}^{L_{j}} \lambda_{j}(t) d t\right)^{n_{j}}}{n_{j} !} \cdot\left\{n_{j} \cdot D_{j}\left(C_{2}^{j}-C_{1}^{j}\right)\right\}
$$

Now considering all the failure modes and the costs for all CMS systems, the expected system cost saving can be expressed as:

$$
\begin{aligned}
E\left[C_{s}\right] & =E\left(\sum_{j=1}^{n} C_{S}^{j}\right)-\sum_{i=1}^{k} C_{C M S}^{i} \\
& =\sum_{j=1}^{n} \sum_{n_{j}=1}^{\infty} \frac{e^{-\int_{0}^{L} \lambda_{j}(t) d t}\left(\int_{0}^{L_{j}} \lambda_{j}(t) d t\right)^{n_{j}}}{n_{j} !} \cdot\left\{n_{j} \cdot D_{j}\left(C_{2}^{j}-C_{1}^{j}\right)\right\}-\sum_{i=1}^{k} C_{C M S}^{i} \\
& =\sum_{j=1}^{n} \sum_{n_{j}=1}^{\infty} \frac{e^{-\int_{0}^{L} \lambda_{j}(t) d t}\left(\int_{0}^{L_{j}} \lambda_{j}(t) d t\right)^{n_{j}}}{n_{j} !} \cdot\left\{n_{j} \cdot\left(1-\prod_{i=1}^{k}\left(1-D_{i j}\right)\right)\left(C_{2}^{j}-C_{1}^{j}\right)\right\}-\sum_{i=1}^{k} C_{C M S}^{i}
\end{aligned}
$$

Note that when multiple CMS systems and multiple failure modes are involved, the aforementioned breakeven point becomes a multidimensional break even surface (BES), which can hardly be obtained as there are multiple detectability variables involved. For the 
demonstration purpose, the following mathematical example with one CMS and two failure modes is used to show the cost benefit analysis discussed above, where the BES between the expected cost saving and two detectability levels are constructed. Assume two failure modes with failure rates equal to 0.333 and 0.5 respectively. The maintenance costs for these two failure modes are $\$ 5,000$ and $\$ 8,000$ respectively, whereas the failure costs are $\$ 20,000$ and $\$ 25000$. The costs for installing a CMS is considered as same with the examples discussed in the previous subsections, which is $\$ 15,000$. Table 3 details the parameter value used in this mathematical example. Figure. (6) represents the expected maintenance cost savings with respect to the detectability levels of the CMS for two different failure modes.

Table 3: Model C Parameters

\begin{tabular}{lllll}
\hline \hline$\lambda$ & $C_{\mathrm{m}}$ & $C_{f}$ & $C_{C M S}$ & $L$ \\
\hline 0.333 & 5,000 & 20,000 & 15,000 & 20 \\
0.5 & 8,000 & 25,000 & 15,000 & 20 \\
\hline \hline
\end{tabular}

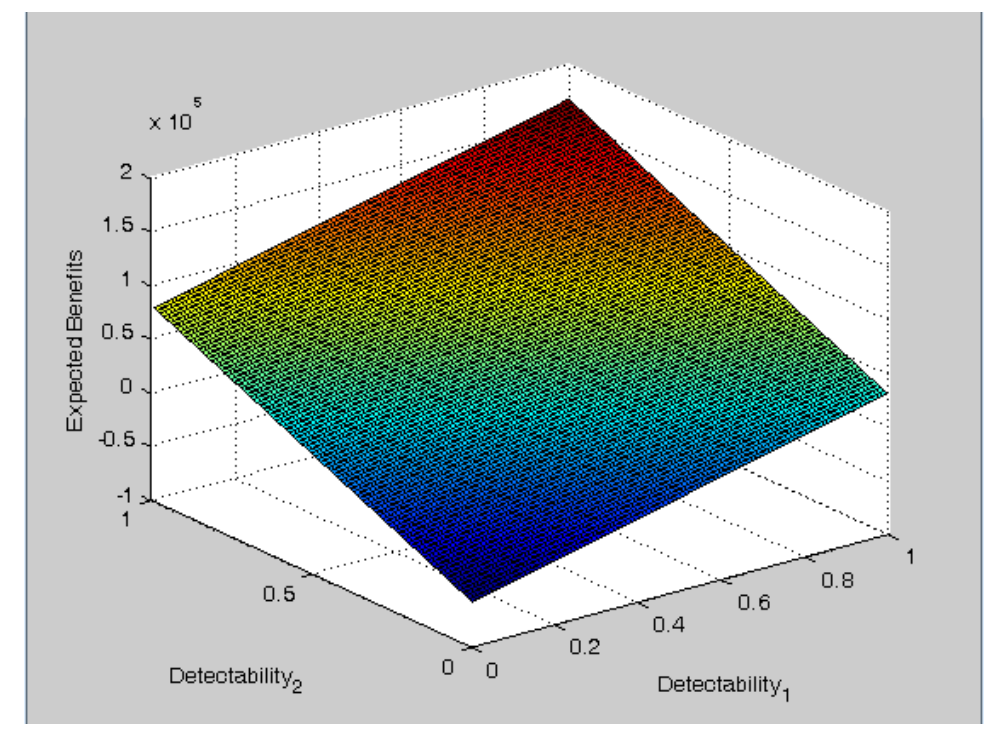

Figure 6. CBA for CMS Results with Two Failure Modes 


\subsection{Multiple Failure Modes Considering CMS System Failures}

In the previous discussion, the failure of the CMS system has not been considered. The failure rate of a CMS system usually also depends on time, with the rate varying over the life cycle of the CMS system. Based on the discussion in Sections II.A and II.B, assuming that the $\mathrm{i}^{\text {th }}$ CMS system has a failure rate function $C M S^{i(t)}$, with this failure rate function, the probability of having $n_{i}$ number of CMS system failures for the $\mathrm{i}^{\text {th }}$ CMS system during the design life $\mathrm{L}$ of a system follows a Non-Homogenous Poisson Process and can be evaluated as:

$$
P_{n_{i}}(t)=\frac{e^{-\int_{0}^{L} \lambda_{\text {CMS }}{ }^{i}(t) d t}\left(\int_{0}^{L} \lambda_{\text {CMS }}{ }^{i}(t) d t\right)^{n_{i}}}{n_{i} !}
$$

As the total number of discrete failure events, $n_{i}$, for the $i^{\text {th }}$ CMS system over the design life of a system is a random variable according to Eq.(6), it is convenient to carry out the expected total CMS systems cost considering failures for all CMS systems, which can be expressed as

$$
E\left[C_{M C S}\right]=\sum_{i=1}^{k} \sum_{n_{i}=1}^{\infty} \frac{e^{-\int_{0}^{L} \lambda_{C M S}{ }^{i}(t) d t}\left(\int_{0}^{L} \lambda_{C M S}{ }^{i}(t) d t\right)^{n_{i}}}{n_{i} !} \cdot\left\{n_{i} \cdot C_{C M S}{ }^{i}\right\}
$$

With the total number of CMS system costs being a random variable as shown in Eq. (17), the total expected system maintenance cost savings based on Eq. (15) can be revised as

$$
\begin{aligned}
E\left[C_{s}\right]= & E\left(\sum_{j=1}^{n} C_{S}^{j}\right)-E\left(C_{C M S}\right) \\
= & \sum_{j=1}^{n} \sum_{n_{j}=1}^{\infty} \frac{e^{-\int_{0}^{L} \lambda_{j}(t) d t}\left(\int_{0}^{L_{j}} \lambda_{j}(t) d t\right)^{n_{j}}}{n_{j} !} \cdot\left\{n_{j} \cdot\left(1-\prod_{i=1}^{k}\left(1-D_{i j}\right)\right)\left(C_{2}^{j}-C_{1}^{j}\right)\right\} \\
& -\sum_{i=1}^{k} \sum_{n_{i}=1}^{\infty} \frac{e^{-\int_{0}^{L} \lambda_{C M S}^{i}(t) d t}\left(\int_{0}^{L} \lambda_{C M S}{ }^{i}(t) d t\right)^{n_{i}}}{n_{i} !} \cdot\left\{n_{i} \cdot C_{C M S}{ }^{i}\right\}
\end{aligned}
$$




\section{CHAPTER 5}

\section{COST BENEFIT ANALYSIS CASE STUDY}

In this section, the developed cost benefit analysis approach will be demonstrated with a three failure modes two condition monitoring systems case study, in which the failures of the condition monitoring systems are also considered. The model parameters, including the failure distributions and the associated costs, for the failure modes and the condition monitoring systems are shown in Table 4. The detectabilities for the two CMS towards the three failure modes are considered from zero to one in order to explore the potential cost savings.

Following the discussion in the Section III, the numbers of failure distributions for the three failure modes are shown in Fig. (7). Due to the consideration of the failures of the CMS and the interactions between the overall detectabilities of the failures modes with the contributions from each individual CMS, the cost saving in this study will first consider the cost saving due to reduced number of failures only. As shown in Fig. (8), the expected cost savings for each failure modes are functions of the detectability values for the two condition monitoring systems. Thus, the overall cost saving by introducing the CMS depends on all the detectability values for different failure modes by different CMS. In order to show the contour of the break even surface, the detectability values for the second and the third failure modes are all fixed to 0.01 while only varying the detectability values for the first failure mode by the two CMS systems from 0 to 1 . By doing so, the contour of the expected cost savings of the overall system with respect detectability values of the first failure mode by the two CMS systems can be seen from the Fig. (9). 
With the information of the cost benefit analysis with multiple failure modes and multiple condition monitoring systems, the employment of condition monitoring systems can be optimized so that the overall system maintenance cost savings can be maximized.

Table 4: Model Parameters for the Case Study

\begin{tabular}{|c|c|c|c|c|}
\hline \multirow{2}{*}{ Components } & \multicolumn{2}{|c|}{ Failure Distribution } & \multicolumn{2}{|c|}{ Cost } \\
\hline & Type & Parameters & $\mathrm{C}_{1}$ & $\mathrm{C}_{2}$ \\
\hline Mode 1 & Weibbull & $\alpha=0.5 \quad \theta=3$ & 2,000 & 20,000 \\
\hline Mode 2 & Exponential & $\lambda=0.25$ & 1,000 & 8,000 \\
\hline Mode 3 & Weibbull & $\alpha=0.25 \quad \theta=2.2$ & 4,000 & 50,000 \\
\hline CMS 1 & Exponential & $\lambda=0.1$ & & \\
\hline CMS 2 & Exponential & $\lambda=0.15$ & & \\
\hline
\end{tabular}

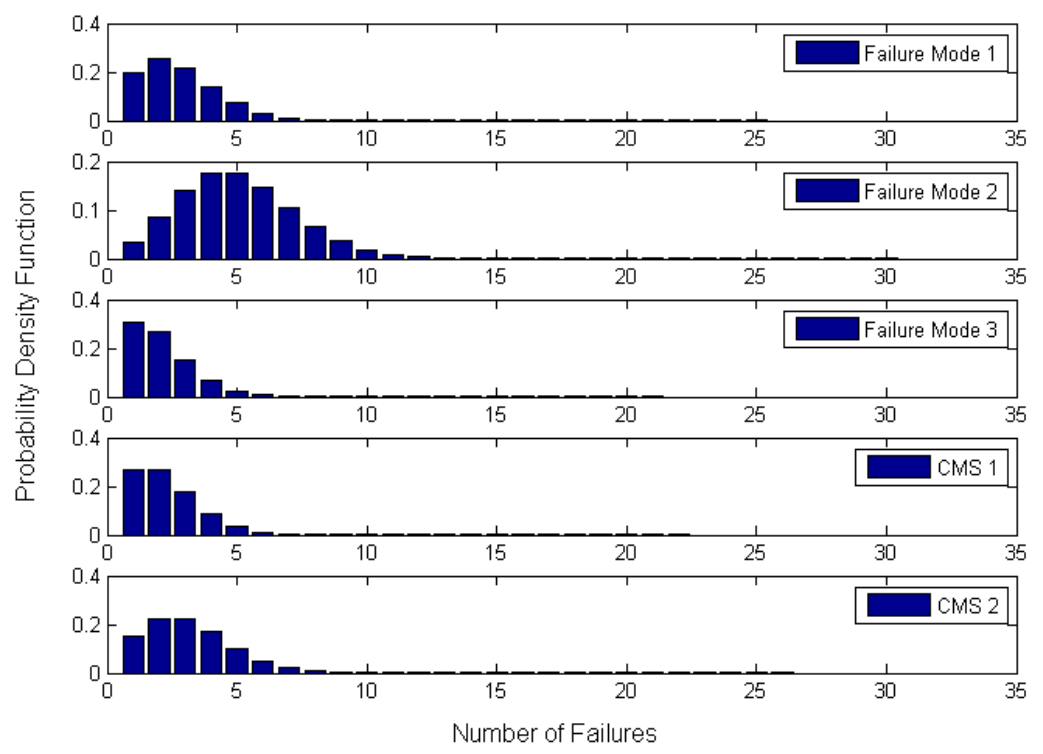

Figure 7. Failures Distributions for Failure Modes and CMS systems 

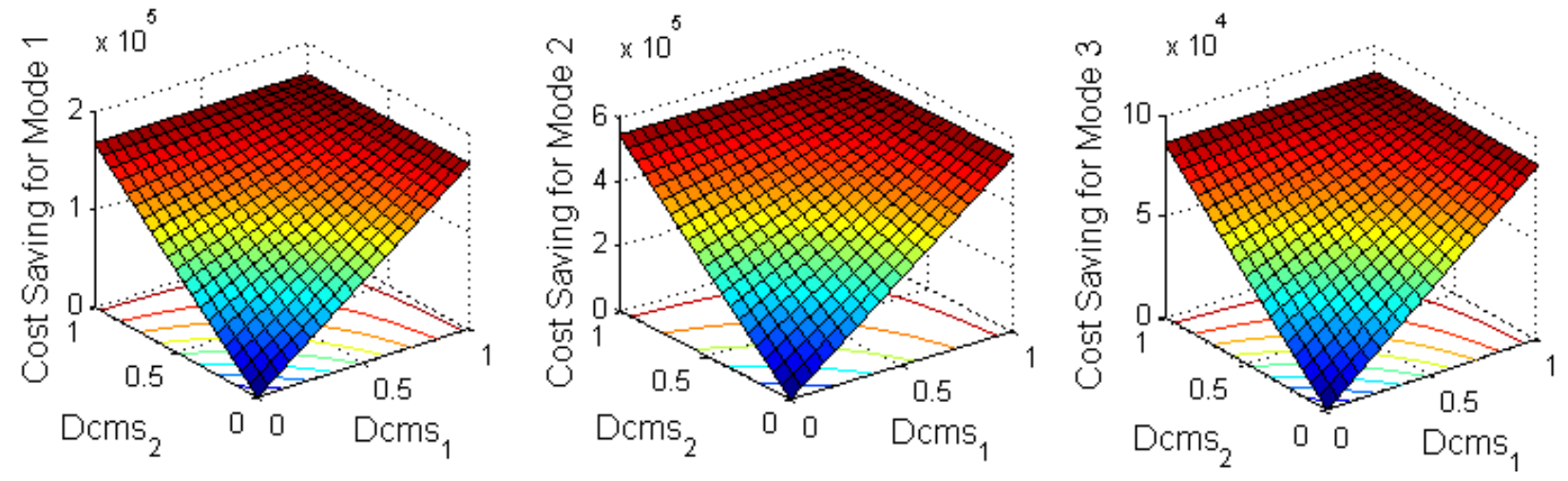

Figure 8. Expected Cost Savings with respect to Detectability Levels from CMS Systems

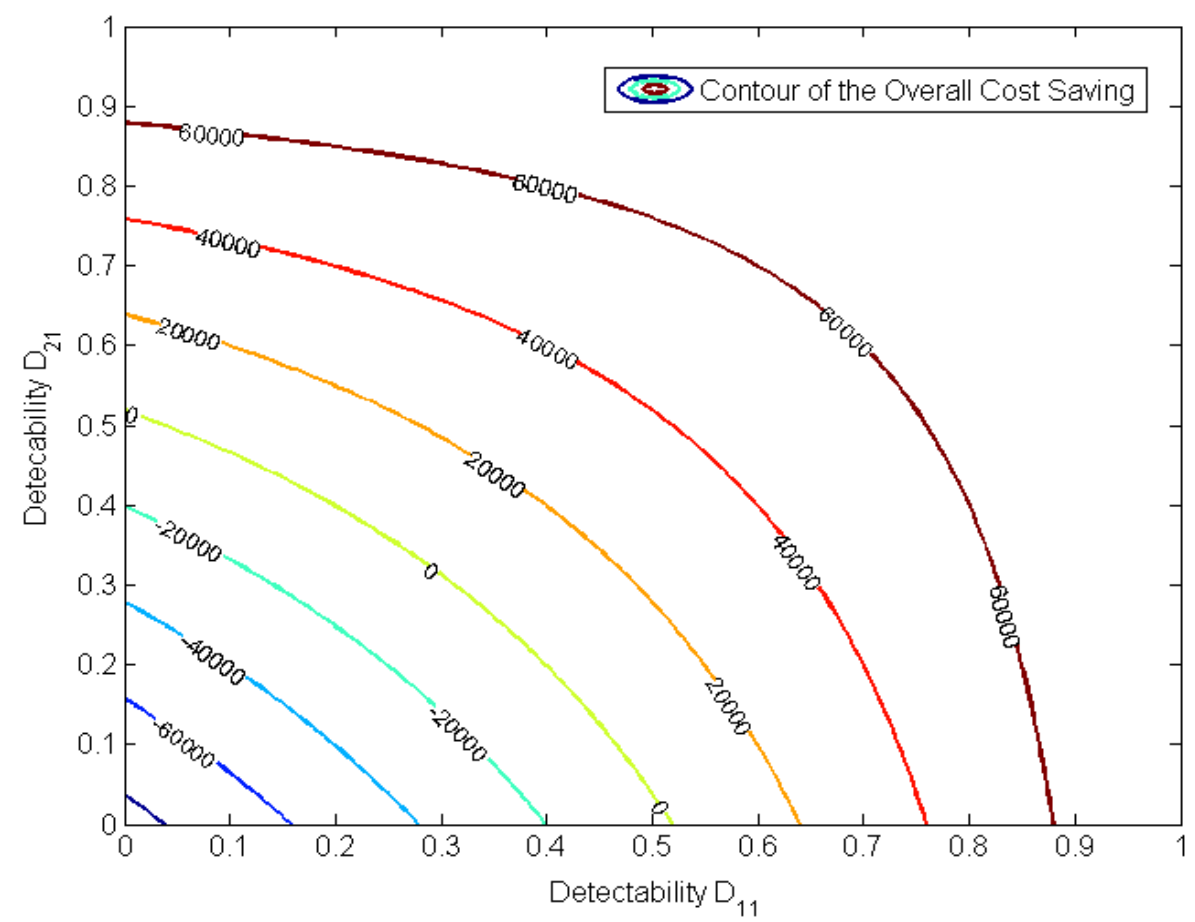

Figure 9. Contour of the Overall Cost Saving with respect to $D_{11}$ and $D_{21}$ 


\section{CHAPTER 6}

\section{CONCLUSION}

This thesis presented a Poisson Process model for the modeling of occurrence of the system-wide failure events and study the potential benefits provided by the CMS in preventing these failure events. With the developed Poisson process model, the cost benefit analysis (CBA) can be implemented by considering the CMS system reliability and costs varying with its failure detection effectiveness presented by the probabilistic detectability measure. Facilitated by CBA of the CMS, break-even points (BEP) or the break-even surface (BES) between expected lifecycle benefits and the required CMS detectability levels can be found to select optimal CMS for different system failure modes. Moreover, with the help of the CBA results, optimal maintenance strategies can be determined to minimize the O\&M costs. The presented CBA methodology for the CMS systems was demonstrated with a maintenance case study and the efficacy was validated. 


\section{REFERENCES}




\section{REFERENCES}

[1] C. E. Ebeling, An Introduction to reliability and maintainability engineering. Long Grove, Illinois: Waveland Press, 2010.

[2] R. Dekker, "Applications of maintenance optimization models: a review and analysis," Reliability Engineering \&amp; System Safety, vol. 51, pp. 229-240, 1996.

[3] T. Licht and A. Deshmukh, "Hierarchically Organized Bayesian Networks for Distributed Sensor Networks," ASME Conference Proceedings, vol. 2002, pp. 1059-1066, 2002.

[4] M. Pecht, "Prognostics and Health Management of Electronics," in Encyclopedia of Structural Health Monitoring, ed: John Wiley \& Sons, Ltd, 2009.

[5] P. Tamilselvan, P. Wang, and B. D. Youn, "Multi-Sensor Health Diagnosis Using Deep Belief Network Based State Classification," ASME Conference Proceedings, vol. 2011, pp. 749758, 2011.

[6] Y. Li, S. Billington, C. Zhang, T. Kurfess, S. Danyluk, and S. Liang, "Dynamic Prognostic Prediction of Defect Propagation on Rolling Element Bearings," Tribology Transactions, vol. 42, pp. 385-392, 1999/01/01 1999.

[7] I. E. Alguindigue, A. Loskiewicz-Buczak, and R. E. Uhrig, "Monitoring and diagnosis of rolling element bearings using artificial neural networks," Industrial Electronics, IEEE Transactions on, vol. 40, pp. 209-217, 1993.

[8] K. F. Martin, "A review by discussion of condition monitoring and fault diagnosis in machine tools," International Journal of Machine Tools and Manufacture, vol. 34, pp. 527-551, 1994.

[9] C. Booth and J. R. McDonald, "The use of artificial neural networks for condition monitoring of electrical power transformers," Neurocomputing, vol. 23, pp. 97-109, 1998.

[10] W. Lu and C. Fulei, "Condition monitoring and fault diagnostics of wind turbines," in Prognostics and Health Management Conference, 2010. PHM '10., 2010, pp. 1-11. 
[11] W. Yang, P. J. Tavner, and M. Wilkinson, "Wind turbine condition monitoring and fault diagnosis using both mechanical and electrical signatures," in Advanced Intelligent Mechatronics, 2008. AIM 2008. IEEE/ASME International Conference on, 2008, pp. 1296-1301.

[12] Z. Daneshi-Far, G. A. Capolino, and H. Henao, "Review of failures and condition monitoring in wind turbine generators," in Electrical Machines (ICEM), 2010 XIX International Conference on, 2010, pp. 1-6.

[13] C. Tsai, H. Cheng-Tao, and H. Shyh-Jier, "Enhancement of damage-detection of wind turbine blades via CWT-based approaches," Energy Conversion, IEEE Transactions on, vol. 21, pp. 776-781, 2006.

[14] S. Yang, L. Wenhai, and W. Canlin, "The intelligent fault diagnosis of wind turbine gearbox based on artificial neural network," in Condition Monitoring and Diagnosis, 2008. CMD 2008. International Conference on, 2008, pp. 1327-1330.

[15] Q. Huang, D. Jiang, L. Hong, and Y. Ding, "Application of Wavelet Neural Networks on Vibration Fault Diagnosis for Wind Turbine Gearbox Advances in Neural Networks - ISNN 2008." vol. 5264, F. Sun, J. Zhang, Y. Tan, J. Cao, and W. Yu, Eds., ed: Springer Berlin / Heidelberg, 2008, pp. 313-320.

[16] M. R. Wilkinson, F. Spinato, and P. J. Tavner, "Condition Monitoring of Generators; Other Subassemblies in Wind Turbine Drive Trains," in Diagnostics for Electric Machines, Power Electronics and Drives, 2007. SDEMPED 2007. IEEE International Symposium on, 2007, pp. 388-392.

[17] Condition Monitoring of Slow Speed Rotating Machinery Using Acoustic Emission Technology. Available: http://dspace.lib.cranfield.ac.uk/handle/1826/4568 [04/01/2013]

[18] P. A. Joosse, M. J. Blanch, A. G. Dutton, D. A. Kouroussis, T. P. Philippidis, and P. S. Vionis, "Acoustic Emission Monitoring of Small Wind Turbine Blades," Journal of Solar Energy Engineering, vol. 124, pp. 446-454, 2002.

[19] E. Wiggelinkhuizen, T. Verbruggen, H. Braam, L. Rademakers, J. Xiang, and S. Watson, "Assessment of Condition Monitoring Techniques for Offshore Wind Farms," Journal of Solar Energy Engineering, vol. 130, p. 031004, 2008. 
[20] J. Nilsson and L. Bertling, "Maintenance Management of Wind Power Systems Using Condition Monitoring Systems\&mdash;Life Cycle Cost Analysis for Two Case Studies," Energy Conversion, IEEE Transactions on, vol. 22, pp. 223-229, 2007.

[21] F. Besnard, J. Nilsson, and L. Bertling, "On the economic benefits of using Condition Monitoring Systems for maintenance management of wind power systems," in Probabilistic Methods Applied to Power Systems (PMAPS), 2010 IEEE 11th International Conference on, 2010, pp. 160-165.

[22] J. J. Nielsen and J. D. Sørensen, "On risk-based operation and maintenance of offshore wind turbine components," Reliability Engineering \&amp; System Safety, vol. 96, pp. 218-229, 2011.

[23] S. Mahalungkar and M. Ingram, "Online and manual (offline) vibration monitoring of equipment for reliability centered maintenance," in Cement Industry Technical Conference, 2004. IEEE-IAS/PCA, 2004, pp. 245-261. 